\title{
Téoros
}

Revue de recherche en tourisme

\section{Les vacances hivernales : une nouvelle approche marketing pour le Québec}

\section{Suzanne Chassé}

Volume 8, numéro 3, novembre 1989

Tourisme hivernal

URI : https://id.erudit.org/iderudit/1080305ar

DOI : https://doi.org/10.7202/1080305ar

Aller au sommaire du numéro

Éditeur(s)

Université du Québec à Montréal

ISSN

0712-8657 (imprimé)

1923-2705 (numérique)

Découvrir la revue

Citer cet article

Chassé, S. (1989). Les vacances hivernales : une nouvelle approche marketing pour le Québec. Téoros, 8(3), 8-10. https://doi.org/10.7202/1080305ar d'utilisation que vous pouvez consulter en ligne.

https://apropos.erudit.org/fr/usagers/politique-dutilisation/ 


\section{Les vacances hivernales: Une nouvelle approche marketing pour le Québec}

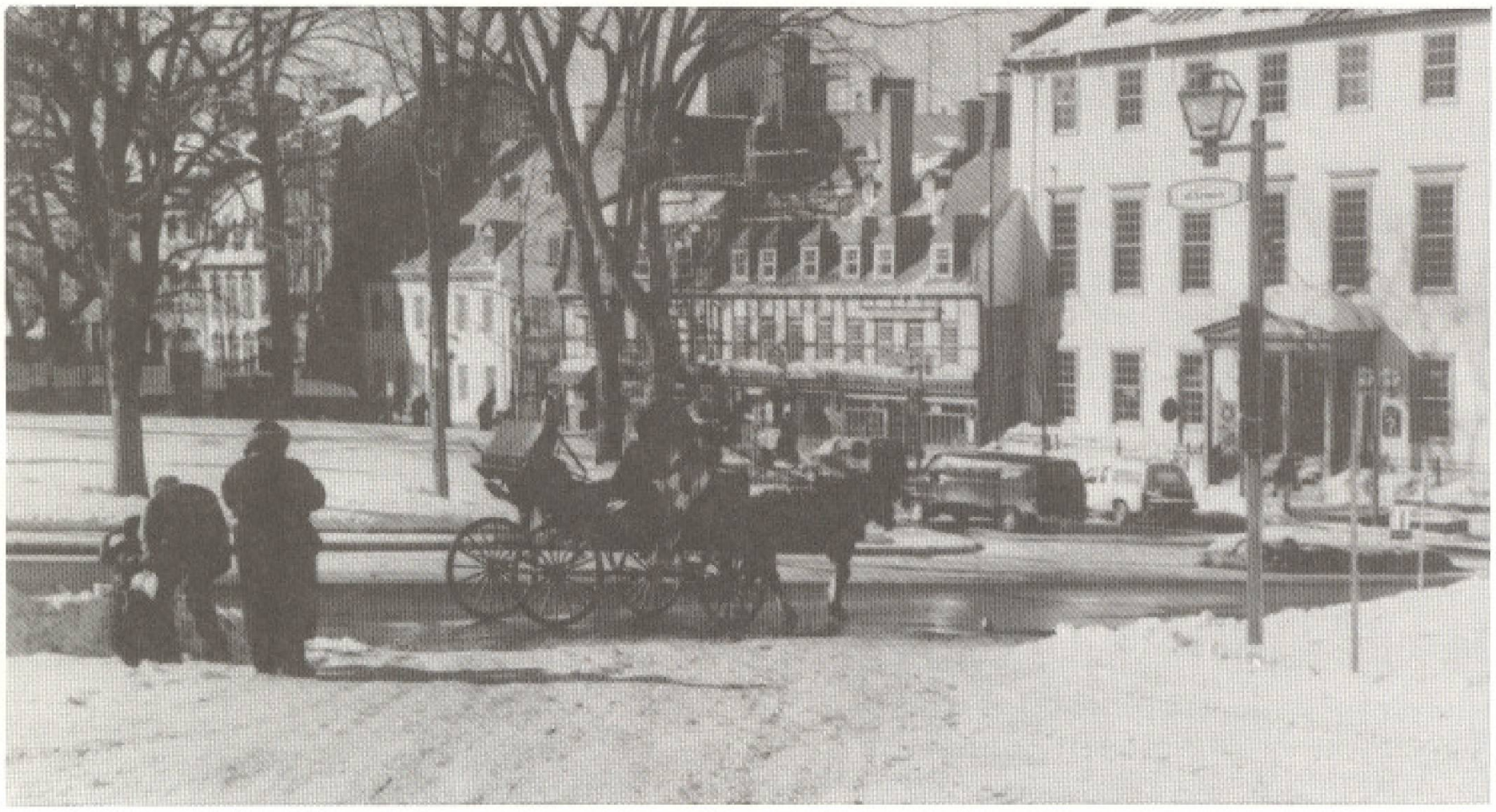

Au printemps 1988, le ministère du Tourisme a entrepris, en collaboration avec le Cabinet Samson Bélair, une démarche de planification stratégique de marketing du tourisme québécois pour une période de trois ans, soit de 1989 à 1992.

Cette démarche a permis d'établir un diagnostic faisant le constat d'un niveau de stagnation des retombées économiques pour le Québec depuis 1980. Pour corriger cet état de fait, les orientations de marketing préconisent des axes stratégiques de concentration, de renforcement et d'alliance. Elles suggèrent aussi de mettre davantage l'accent sur le processus d'achat du consommateur.

Pour y arriver, le ministère du Tourisme chapeautera ses actions sous trois volets:

- Suzanne Chasse est enseignante en tourigme au Collège de Granby et consultante en tourisme. Elle a participe avec le Cabinet Samson Bélair au mandat de planification stratégique du tourisme quebeccois 1989 1992. agrément-été, agrément-hiver et affaires. Sous chacun de ces volets, on retrouvera différents produits. Sous le volet agré= ment-hiver, c'est toute la politique touchant les vacances hivernales au Québec que l'on retrouve. C'est toute une série d'expériences de vacances que l'on offre aux consommateurs et non plus seulement l'activitế ski alpin.

Cette approche par produit isole huit prétextes à un séjour au Québec. Le vocable "produit" signifie qu'il y a huit activités majeures justifiant un déplacement au Québec. Un produit peut être commercial sous le volet agrément-été ou sous le volet agrément-hiver; ces produits sont pour le Québec: les grandes villes, le circuit, le séjour, les congrès, le ski alpin, la chasse et la pêche, la motoneige et l'aventure. Cette gamme de produits s'adresse autant à une clientèle québécoise qu'à une clientèle de l'extếrieur du Québec. C'est dans le positionnement et dans le choix de l'offre de ces produits que l'on différenciera la clientèle. Il va de soi qu'un produit que l'on identifie comme produit d'appel est lié à des produits d"appoint; il est en réalité la pointed'un iceberg relativement complexe.

\section{Produits retenus pour des vacan- ces d'hiver au Québec}

Parmi les huit produits retenus pour le Québec, le ministère du Tourisme en commercialisera cinq durant la période hivernale, soit le ski alpin, la motoneige, le séjour, les grandes villes et l'aventure.

Le ski alpin constitue depuis quelques années un produit d'appel pour le Québec. $\mathrm{C}^{\prime}$ est un produit de base, un excellent vendeur pour une saison à l'origine très déficitaire. De plus, il est bien intégré à la réalité fondamentale du Québec; l'hiver, qui servira toujours de moteur à la vente de la gamme des produits hivernaux. Les investissements ênormes réalisés ces dernières années, tant au niveau des infrastructuress que de celui de la promotion de celte 
activité auprès des clientèles extérieures, démontrent qu'une approche par produit, intégrant toutes les étapes de la mise en marché, est payante. Tout le monde en conviendra, le ski en est au stade de la rentabilité.

La motoneige est une activité qui a été liếe davantage à la pratique de loisir, mais depuis quelques années, elle passe de la récréation au séjour touristique. La motoneige a l'avantage de drainer avec elle l'identité du Québeccomme le ski de fond aété à l'origine identifié à la Scandinavie. Cette activitế se pratique, entre autres, dans des zones de grande nature et permet aux régions de relancer leur chiffre d'affaires dans une saison souvent très déficitaire.

Le séjour est une activité plus complexe car plusieurs activités peuvent justifier un déplacement dans un lieu de villégiature ou dans une station.

Mais un point demeure commun à toutes ces activités: l'hébergement qui rassemble les différents besoins des consommateurs. Le séjourest défini comme une combinaison de formes $\mathrm{d}^{\dagger}$ hébergement (auberge, base de plein air, "resort" de villégiature) à des activités de plein air et/ou des activités culturelles et/ou des activités de mise en forme ou de santé.

Dans un contexte de vacances hivernales, c'est davantage le ski de randonnée qui justifiè le déplacement, mais il se doit d'être complété par des activités telles que la pêche blanche (peche sous glace), le patinage, les promenades en traîneaux, la raquette et des activités liées à la mise en forme (sauna, massage, etc.). Dans un contexte régional, plus il y a d'activites récréo-touristiques associées à l'hébergement, plus la durée de séjour pourra être allongée et répétitive.

Les grandes villes sont aussi une raison de venir au Québec durant la saison hivernale. Montréal, Québec, Hull-Ottawa ont beaucoup à offrir aux touristes (activités culturelles et sportives, magasinage, etc.), surtout depuis que les hôteliers ont mis de l'avant une politique de prix intéressante. Les grandes villes demeurent le meilleur vendeur pour le Québec; elles conviennent bien à la tendance du fractionnement des vacances ( 3 ou 4 jours), elles peuvent accentuer leur attrait via les grands evenements d'hiver (Carnaval de Québec, Bal de Neige et Fête des Neiges) et elles peuvent aussi $s$ 'accompagner $d^{4} u n$ rayonnement en région.
L'aventure est aussi un produit à positionner à l'avant-garde de la gamme des produits du Québec, comme élément d'appel. L'aventure est dotée d'un aura qui canalise l'attention, même si elle est réservét à une clientèle plus limitée. Elle est basée en grande partie sur la randonnée. Elle est liée à un besoin de "sortir de l'ordinaire". C'est un produit vitrine attrayant pour la clientèle car on $y$ retrouve des activités telles que des randonnées de traîneaux tirées par des chiens, de l'héli-ski dans les monts Chic-Chocs, du trekking ou raid en ski de randonnée et en motoneige dans la toundra...

Voilà donc les cinq produits retenus par le ministere du Tourisme pour positionner une expérience de vacances hivernales au Québec. C'est une nouvelle approche marketing qui répond davantage aux besoins des différentes clienteles.

\section{Positionnement du Québec et marchés cibles}

Dans unenvironnement aussi concurrentiel que celui du tourisme, la différence devient la clé de voutte pour une destination. Un de ces avantages différentiels pour le Québec, c'est justement sa saison hivernale. Le ministère du Tourisme doit donc créer dans la tête du consommateur, une image forte pour le convaincre que des vacances dans la neige seraient une expérience intéressante. Compte tenu des différents marchés auxquels ils s'adressent, certains produits répondront davantage aux attentes des consommateurs.

Face aux marchés ontarien et américain. s ajouteront le caractère distinctif du Québec et sa spécificité culturelle aux activités hivernales de ski alpin et de motoneige. A l'outre-mer, c'est davantage les grands espaces, la grande nature et l'aventure qui fascinent le touriste. Il recherche l'aspect spectaculaire des grandes étendues. Ce positionnement est d'autant plus nécessaire car le Québec est perçu comme une partie du Canada.

Quant à la clientèle québécoise, elle sera toujours attirée par des destinations soleil, le Québécois ayant besoin de chaleur et d'exotisme pendant la période hivemale. Malgré ce constat, les tendances observées du fractionnement des vacances, le comportement d'acteur plutôt que de spectateur incite la clientèle québécoise à vivre différentes expériences dont celles de vacances hivernales au Québec. C'est la diversité, le plaisir lié aux activités de plein air, à la gastronomie, ete., qui seront mis de l'avant dans ce cadre de vacances.

Pour tous les marchés, c'est la complémentarité des activités et les multiples possibilités de combinaisons qui permettront d'adapter des vacances hivernales aux diffêrents besoins de la clientèle.

\section{Mise en marché des différents produits}

Afin de compléter plus spécifiquement la planification stratégique globale, des plans de marketing par produit ont ếté élaborés par le ministère du Tourisme. Compte tenu du niveau de développement de chacun des produits, le plan marketing de certains d'entre eux est plus poussé que d'autres; le ski alpin à titre d'exemple, est un produit sur lequel beaucoup d'études ont étế complétées. On retrouve donc dans ces plans de marketing par produit, d'abord l'identification des clienteles cibles et par la suite, des stratégies liées au positionnement, aux arguments de ventes, à la production de brochures, aux campagnes promotionnelles, à la présence du Québec lors de "market-place" ou de foires, à la distribution, au service à la clientèle, etc.

Pour chacun des produits retenus dans le cadre de vacances hivernales au Québec, identifions maintenant quelques stratégies suggerées dans le rappont Samson Bélair. Dans le cadre de l'activité ski alpin, le ministère du Tourisme devrait travailler avec les neuf stations majeures telles qu'identifiées par le ministère du Loisir, de la Chasse et de la Pêche. Ces stations se répartissent dans trois régions du Québec, soit l'Estrie, les Laurentides et la région de Québec. Enplusd'identifier les spécificités offertes au niveau de la pratique du ski, l'on devrait mettre davantage l'accent sur l'encadrement de qualité de cette activité (École de ski, navette, etc.) et de la qualité de l'enneigement. Le ski de soirée peut aussi devenir un élément intéressant de vente illustrant l'avantage différentiel. La possibilité de pouvoir skier dans différents centres est un atout grâce aux formules développées dans les régions. Quant au processus $d^{4}$ achat, il est important de mettre en valeur l'eventail de forfaits disponibles.

Lorsque $I^{\dagger}$ on choisit de venir skier au Québec (après s'être assuré de la variété et du nombre de pentes, de remontées mécaniques, etc.), on le fait non seulement pour la valeur du ski, mais aussi en tenant compte des éléments qui nous distinguent des autres destinations (gastronomie, accueil, etc.). 


\section{ILLSUTRATION DE LA DEFFINITION D'UN PRODUIT EXEMPLE CHOISI: LE SÉJOUR}

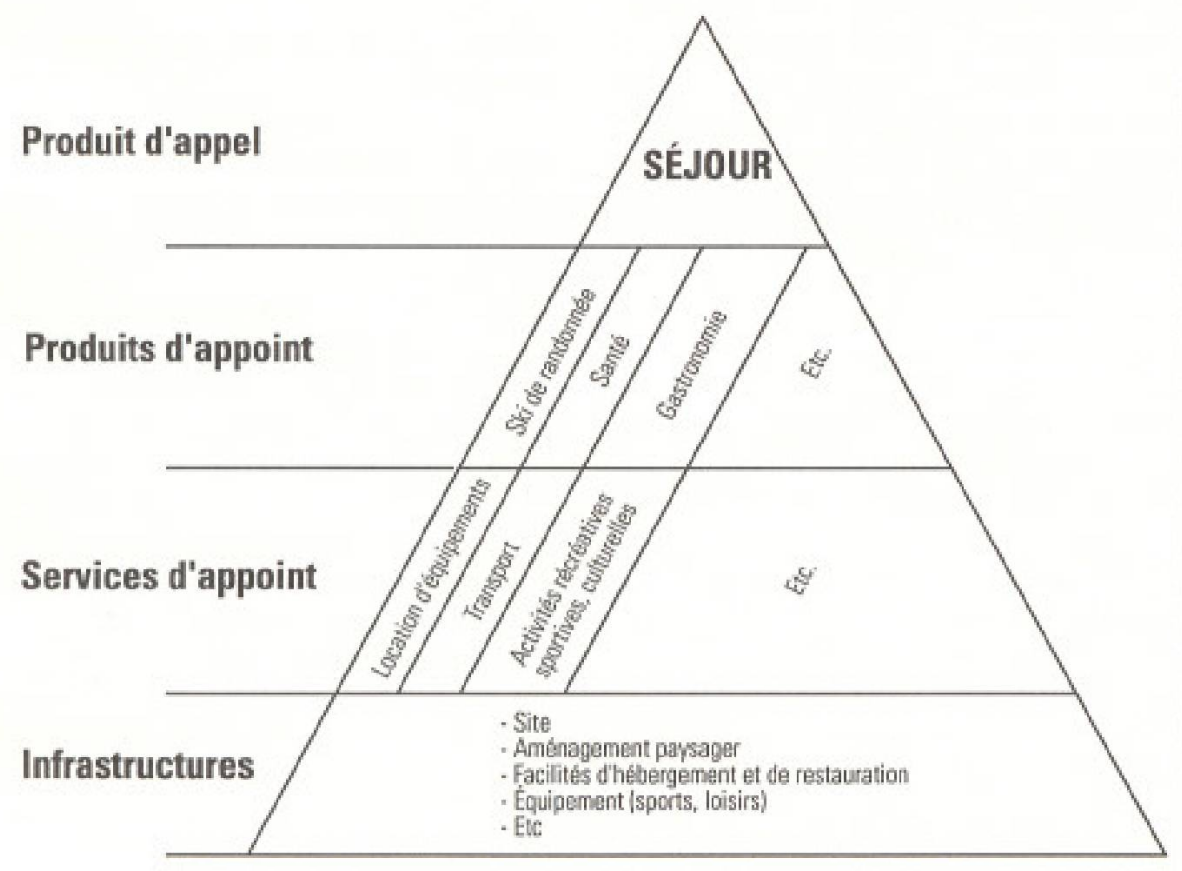

C'est sur ces avantages différentiels qu'il faut tabler.

Quant à la mise en marché de l'activité motoneige, c'est sous une double approche que l'on pourrait travailler. D'abord pour les initiés, mettre en évidence le réseau Trans-Québec de 8000 kilomètres de pistes balisées offrant une grande diversité de parcours, interreliés entre eux et avec un accès direct à des services d'hébergement, de restauration, d'essence et d'entretien.

D'autre part, pour les non-initiés, faire connaître les forfaits disponibles qui intègrent la location de véhicules, de vêtements, etc. Cette activité jointe à d'autres, permettraient de vivre des vacances hivernales diversifiées. De plus, la promotion d'événements positionnera le Québec comme destination auprès des motoneigistes.

C'est avec le produit séjour que le concept de vacances hivernales prend toute sa signification. C'est l'addition de toute une série d'activités possibles qui rénd le séjour attrayant auprès du consommateur. C'est un peu la stratégie de buffet qui est utilisée; le touriste ne pratiquera probablement pas toutes les activités offertes mais il sait qu'il peut choisir au gré de sa fantaisie entre le ski de randonnée, le patinage, la pêche sous la glace, le tobbogan et le ski alpin. À cela s'ajoute une préoccupation de service à la clientèle où le séjour du visiteur devient l'élément primordial de ses hôtes. L'hospitalité québécoise, le caractère distinctif du Québec et la gastronomie servirontd' arguments de vente auprès d'une clientèle extérieur au Québec.

Un autre produit, soit celui des grandes villes représentent aussi une expérience de vacances intéressante en hiver. Comme pour toutes grandes villes du monde, c'est la saison où il $\mathrm{y}$ a la plus grande densité d'activités culturelles. C'est d'ailleurs le caractère distinctif du Québec qu'il faut mettre de l'avant. Ce caractère que l'on retrouve dans la restauration, dans les musées et même dans le magasinage.

Deux autresélémentss'ajoutent: d'une part, à l'intérieur même de ces villes, il est possible de pratiquer des activités de plein air (ski de randonnéce et patinage) et $\mathrm{d}^{4}$ autre part, chacune aun événement majeur offrant un programme d'activités complémentaires, créant ainsi une animation plus dense.

Le dernier produit, soit le tourisme d'aventure ne doit pas être associé exclusivement à un risque élevé. On peut offrir cette activité en dosant le danger et en tenant compte des clientèles auxquelles on s'adresse. C'est ici que le terme "nordicité" prend toute sa saveur. Pour y arriver, toute une combinaison d'activités sont possibles et la participation des autochtones aux différents forfaits crée un intérêt supplémentaire (Amérindiens et Inuits).

La présence du Québec dans des salons consacrés aux produits d'aventure, la possibilité de rencontre entre les grossistes réceptifs et la clientèle potentielle (afin de les sécuriser), la couverture médiatique autour d'évếnements (ex.: "Haricana", le Paris-Dakarde la motoneige) sont autant de techniques de mise en marché pour positionner ce produit, mais aussi pour positionner la destination et les vacances hivernales au Québec.

\section{Conclusion}

L'offre trop atomisée et la mise en marché tropéparpillée et dotée de moyens financiers relativement faibles obligent le Québec àse doter d'une nouvelle approche marketing.

Deux objectifs devront être respectés afin de maximiser les retombées économiques: l'efficacité et l'adaptation de l'offre à la demande!

Afin $d^{*} y$ arriver, le ministère du Tourisme privilégie uneapproche globale du tourisme hivernal. Le ski alpin a toujours une place prépondérante, mais il n'est plus le seul produit d'appel pour une telle saison.

Le ministère du Tourisme s'est donc donné un plan d'action général pour le secteur agrément-hiver. Dans un premier temps, il a établi un volet institutionnel, c'est-à-dire qu'il a défini des actions relevant exclusivement de son intervention (publicité, outils promotionnels, etc.) et dans un second temps, il a établi un volet associatif où il n'est plus le seul intervenant, mais où il travaille en collaboration avec l'entreprise privée.

La démarche pour coordonner cette approche, a amené le ministère du Tourisme à identifier des "chefs de produit" qui ont pour mandat d'assurer une continuité entre le besoin du consommateur, 1 'offre du Québec et les actions nécessaires pour favoriser l' achat de ce produit. 